\title{
Enhanced Magnetization in a Frustrated Spin System by Ni+ Ion Beam Irradiation
}

\section{Jun Kue Park ( $\nabla$ jkuepark@kaeri.re.kr)}

Korea Multi-purpose Accelerator Complex, Korea Atomic Energy Research Institute

\section{Hye Min Jang}

Korea Multi-purpose Accelerator Complex, Korea Atomic Energy Research Institute

Won-Je Cho

Korea Multi-purpose Accelerator Complex, Korea Atomic Energy Research Institute

Chorong Kim

Korea Multi-purpose Accelerator Complex, Korea Atomic Energy Research Institute Jaekwon Suk

Korea Multi-purpose Accelerator Complex, Korea Atomic Energy Research Institute

\section{Dong-Seok Kim}

Korea Multi-purpose Accelerator Complex, Korea Atomic Energy Research Institute Jae Sang Lee

Korea Multi-purpose Accelerator Complex, Korea Atomic Energy Research Institute

\section{Research Article}

Keywords: carbonyl iron (Cl), Ni+ ion beam, magnetization, zero-field cooled (ZFC), irradiation

Posted Date: July 28th, 2021

DOI: https://doi.org/10.21203/rs.3.rs-745911/v1

License: (c) (i) This work is licensed under a Creative Commons Attribution 4.0 International License.

Read Full License 


\title{
Enhanced magnetization in a frustrated spin system by $\mathrm{Ni}^{+}$ion beam irradiation
}

\author{
Jun Kue Park ${ }^{1, *}$, Hye Min Jang ${ }^{1}$, Won-Je Cho ${ }^{1, \dagger}$, Chorong Kim ${ }^{1}$, Jaekwon Suk ${ }^{1}$, \\ Dong-Seok Kim ${ }^{1}$, and Jae Sang Lee ${ }^{1}$
}

\author{
${ }^{1}$ Korea Multi-purpose Accelerator Complex, Korea Atomic Energy Research Institute, Gyeongju 38180, Korea \\ *jkuepark@kaeri.re.kr \\ †wonje59@kaeri.re.kr
}

\begin{abstract}
We investigate the magnetic properties in a frustrated spin system of carbonyl iron $(\mathrm{Cl})$ particles before and after $\mathrm{Ni}^{+}$ion beam irradiation. Upon increasing temperatures, the saturation magnetization exhibits an anomalous increase, which is more intense after the beam irradiation. The zero-field cooled (ZFC) magnetization data show an anomalous increase up to $300 \mathrm{~K}$, regardless of the beam irradiation. After the irradiation, unlike in the unirradiated $\mathrm{CI}$ particles, the ZFC curve shows separated regimes, reflecting two distributions of the blocking temperature, which may be related to the particle distribution summed with two distribution functions. After the irradiation, strong interparticle interaction may be present due to the effect of dipolar interaction among $\mathrm{Cl}$ particles doped $\mathrm{Ni}$ ions, leading to the enhanced magnetization. We may suggest that the anomalous magnetization behavior can be ascribed to frustration in the internal magnetic order for the unirradiated $\mathrm{Cl}$ particles, and further interparticle interaction for the irradiated $\mathrm{Cl}$ particles.
\end{abstract}

\section{Introduction}

Carbonyl iron (CI) powders are well known as microwave absorption materials due to their superior electric conductivity, high saturation magnetization, high Curie-temperature, and wide absorption bandwidth of $2-18 \mathrm{GHz} .{ }^{1-8}$ In particular, a relatively high density and a high filling content make them more proper in electromagnetic wave absorption materials. From a point of view in magnetic properties, CI powders can be applied to magnetorheological suspensions where their favorable magnetic properties change Newtonian or pseudoplastic fluids in the absence of magnetic field to viscoplastic solid in the presence of magnetic field, creating chain-like structures along a direction of the field. ${ }^{4,5}$ Their superior controllability of the viscosity in the systems may thus successfully be exploited in industrial applications.

Oxidation process in CI particles may affect magnetization properties in which the saturation magnetization $\left(M_{\mathrm{s}}\right)$ shows a decrease with oxidation, leading to the formation of maghemite $\left(\mathrm{Fe}_{2} \mathrm{O}_{3}\right){ }^{5}$ A decrease of $M_{\mathrm{S}}$ and an increase of the saturation field may arise from a decrease of the Ruderman-Kittel-Kasuya-Yosida interactions. Previously, Pan etal. reported that the particle morphology such as spherical and flaky shapes may also affect the magnetic properties and microwave absorption properties, due to the different magnetic loss mechanisms depending on the particle shape. ${ }^{3}$ Also, particle size is one of the main factors in changing magnetic properties. ${ }^{3,4,6,9,10}$ The distribution of the size may result in a distribution of the so-called blocking temperature, above which an unstable magnetization may establish due to thermal fluctuations. ${ }^{6}$

Although there are many studies to investigate the electromagnetic wave absorption properties of CI powder, ${ }^{1-4,7,8}$ a comprehensive study for the magnetic properties of CI powders has not been reported so far. Moreover, a few studies reported on the understanding of the properties resulting from doping of transition metal ions, in particular. In magnetic particles, one of the important magnetic issues is a nonlinear spin configuration in the surface region, from which surface spin-glass behavior takes place. The spin-glass-like phase with a multidegenerate ground state may be due to competing magnetic interactions arising from the combination of structural disorder and frustration. Previously, in oxidized Fe nanoparticles, a critical diameter may trigger an abrupt surface spin-glass behavior and exchange bias effect. ${ }^{6}$ Interface effects leading to multiphase character may give rise to intraparticle exchange anisotropy. In addition, the presence of magnetic interactions between particles can increase the magnetization, modifying the surface configuration of spins. ${ }^{9}$ In this paper, Ni-doped CI particles were obtained from $\mathrm{Ni}^{+}$ion beam irradiation. We observed an anomalous increase in $M_{\mathrm{S}}$ at elevated temperature for the unirradiated sample, and an intensified $M_{\mathrm{S}}$ was observed after $\mathrm{Ni}^{+}$ion beam irradiation. We display the results of dc magnetic properties with respect to temperature and magnetic field for the samples before and after the beam irradiation, showing evidence of the existence of frustration in the internal magnetic order of the particles and magnetic interactions between the particles. 


\section{Results and Discussion}

Figure 1(a) shows the X-ray diffraction (XRD) patterns for CI powders before and after $\mathrm{Ni}^{+}$beam irradiation. Three main diffraction peaks correspond to single cubic $\alpha$-Fe (PDF\#06-0696). ${ }^{1,3,4,11}$ In both the samples, no other peaks due to impurities are detected. The inset of Fig. 1 shows the scanning electron microscopy (SEM) image of the unirradiated CI, in which the size and morphology of the particles were observed. The particles are spherical with the smooth surface in shape, hindering the formation of spherical agglomerates. ${ }^{3,11}$ It is thus expected that the uniform dispersion of CI powders in the matrix. In Fig. 1(b) and 1(c), the Fe 2p X-ray photoelectron spectrometry (XPS) spectra for CI powders before and after irradiation. The spectra were fitted well with four Gaussian lines after the Shirley background was subtracted. The peaks at $711.3 \mathrm{eV}$ and $725.3 \mathrm{eV}$ were ascribed to the $\mathrm{Fe}^{3+} 2 \mathrm{p}_{2 / 3}$ and $\mathrm{Fe}^{3+} 2 \mathrm{p}_{1 / 2}$ electrons, respectively. ${ }^{4,5,12}$ The binding energies at $707 \mathrm{eV}$ and $719 \mathrm{eV}$ would correspond to the metallic Fe state, but they do not seen in our data. Taking into account of the presence of $\mathrm{O} 1 \mathrm{~s}$ peak in the survey spectra, we may suggest that some Fe elements on the surface of the samples before and after irradiation are oxidized to $\mathrm{Fe}_{2} \mathrm{O}_{3}$. Using the SRIM2008 package, we simulated the mean implantation depth and profile of $\mathrm{Ni}^{+}$beam with $140 \mathrm{keV}$ onto CI. The calculated depth was about $46 \mathrm{~nm}$. From the inductive coupled plasma mass spectroscopy (ICP-MS) elemental analysis, we clearly observed ${ }^{58} \mathrm{Ni}$ as much as $3.8 \mathrm{ppb}$ for the irradiated particles, whereas it is below detection limit for the unirradiated particles.

Figure 2 shows the particle size histogram obtained from the dynamic light scattering (DLS) measurement, together with fits by using a log-normal distribution of particle diameters. The distribution may be given by ${ }^{13,14}$

$$
f(D)=\frac{1}{\sqrt{2 \pi} \sigma D} \exp \left(-\frac{\ln ^{2}\left(\frac{D}{\langle D\rangle}\right)}{2 \sigma_{D}^{2}}\right),
$$

where $\langle D\rangle$ and $\sigma_{D}$ denote the median particle diameter and the distribution width, respectively. Before the irradiation, the distribution may be fitted with a single log-normal distribution, as shown in Fig. 2(a). Unlike the unirradiated sample, however, the distribution is markedly changed for the irradiated sample, exhibiting a fit with the two distribution functions (Fig. 2(b)). It is an intriguing characteristic for this changed distribution after the irradiation. Taking into account the method of the suspended particles in DLS, the changed distribution can only be explained by an interaction introduced between the particles.

Figures 3(a) and (b) show magnetization curves of the Fe particles before and after irradiation measured at $5 \mathrm{~K}$ and 300 $\mathrm{K}$, in which the magnetic field swept from $-30 \mathrm{kOe}$ and $30 \mathrm{kOe}$. As shown in the figure, both the samples show the magnetic hysteresis curves with very small similar coercive fields of $H_{c}=\sim 21$ Oe and $H_{c}=\sim 29$ Oe at $5 \mathrm{~K}$ and $300 \mathrm{~K}$, respectively. Intriguingly, the magnetization is greater at a high temperature of $300 \mathrm{~K}$ than at a low temperature of $5 \mathrm{~K}$ in both the sample. It should be also noted that saturation magnetization $M_{\mathrm{S}}$ of the irradiated sample is about two times greater than that of the unirradiated sample, indicating $\mathrm{Ni}^{+}$ions doped into $\mathrm{Fe}$ particles enhance the magnetization. Typically, in superparamagnetic nanoparticles, the magnetic anisotropy on the surface may increase, but in the bulk they may have the ferromagnetic single domain. ${ }^{15-17}$ A superparamagnetic behavior shows an increasing magnetization with increasing applied magnetic field with no remanent magnetization and coercive field in the nanoparticles. However, in our hysteresis curves, the $M_{\mathrm{s}}$ was observed at $\sim 10 \mathrm{kOe}$, which is unlikely to the superparamagnetic behavior. Furthermore, the $H_{c}$ should become to be zero, if the system exhibits a superparamagnetic behavior, which is thus not our case. Taking into account a broad distribution with greater size of our particles $(\sim \mu \mathrm{m})$, we may expect an intraparticle interaction among the domains, which is triggering frustration between magnetically ordered regions within a particle. ${ }^{6,9,18}$ Unlike other $\mathrm{M}-\mathrm{H}$ curves, the magnetization at $300 \mathrm{~K}$ for the irradiated sample shows a slight increase with increasing the field, but not a saturation.

In Figs. 4(a) and (b), the zero-field cooled (ZFC) and field cooled (FC) magnetization curves are displayed. First, we may consider the ZFC curves for both samples. When the particles cooled in zero field, each particle aligns with the random direction of the easy axis due to its magnetic anisotropy energy barrier being enhanced with decreasing temperature. Because of the random orientation of the easy axis, the net magnetization approaches zero at very low temperatures. ${ }^{15,19,20}$ Then, as the particles are warmed up to $300 \mathrm{~K}$ in the presence of the field $(500 \mathrm{Oe})$, they can switch their magnetization direction from the easy axis to the applied field due to thermal energy, leading to an increase in the overall magnetization, as shown in the ZFC curves of Figs. 4(a) and (b). Typically, the ZFC curve in non-interacting single domain particles shows a decrease in the magnetization above the blocking temperature $T_{\mathrm{B}}$, where an assembly of identical single-domain particles goes from blocked to superparamagnetic-type behavior. ${ }^{15,17,21,22}$ It is thus in a superparamagnetic regime at room temperature.

However, in our ZFC data, we cannot observe a decrease in magnetization at elevated temperatures up to $300 \mathrm{~K}$, indicating the system obeys a ferromagnetic behavior rather than superparamagnetic behavior in this temperature regime. Note that for the irradiated sample, the ZFC curve seems to have separated regimes appearing a shallow kink at $\sim 200 \mathrm{~K}$, which is distinct from that in the unirradiated sample where no separated regime appears.

For the FC curves, upon decreasing temperatures under an applied field $(500 \mathrm{Oe})$, the direction of the magnetic moment for each particle will tend to align with the easy axis closest to the applied field direction, and thus remain locked in that 
direction. In other words, the magnetic anisotropy energy barrier increases with reducing temperature, making the magnetic moments align with the direction of the easy axis. In a typical FC curve for a superparamagnetic system, we may thus expect the magnetization of the particles would be increased with decreasing temperatures. ${ }^{19,22,23}$ However, in our FC curves, the magnetization shows a decrease with decreasing temperature, and exhibits no appreciable change below $\sim 200 \mathrm{~K}$, featuring the spin-glass-like behavior. The spin glass behavior arises from intraparticle interaction or magnetic order frustration at the particle surface, enhancing the effective anisotropy. Hence, spin frustration in the internal magnetic order may explain this anomalous behavior in the ZFC and FC curves, giving a reason for why $M_{s}$ is greater at high temperature. Moreover, in our ZFC and FC curves shown in Figs. 4(a) and 4(b), we cannot observe a clear branching point up to $300 \mathrm{~K}$. Above the branching point, the superparamagnetic behavior would be dominant. ${ }^{15,21,24,25}$

Figures $4(\mathrm{c})$ and (d) show the data obtained from the derivative $d\left(M_{\mathrm{ZFC}}-M_{\mathrm{FC}}\right) / d T$ of the experimental data. The decreasing slopes of the distribution were equivalently observed above $3 \mathrm{~K}$ in both samples, but unlike in the unirradiated sample, a peak at $\sim 200 \mathrm{~K}$ was observed in the irradiated samples, indicating a separated regime of $f\left(T_{\mathrm{B}}\right)$ may be present, as was noted in the ZFC curve of Fig. 4(b).

Given that the particles are in the regime of being blocked or superparamagnetic, the blocking temperature $T_{\mathrm{B}}$ can be expressed as, ${ }^{14,15,17}$

$$
T_{\mathrm{B}}=\frac{K V}{\ln \left(\tau_{\mathrm{obs}} / \tau_{0}\right) k_{\mathrm{B}}},
$$

where $K$ is the uniaxial anisotropy constant, $V$ is the particle volume, $\tau_{\mathrm{obs}}$ is an observation time, and $\tau_{0}$ is the characteristic time constant. Taking into account the particle size distribution, we can define the distribution of blocking temperatures, $f\left(T_{\mathrm{B}}\right)$, assuming to be a log-normal distribution, ${ }^{14}$

$$
f\left(T_{\mathrm{B}}\right)=\frac{A}{T_{\mathrm{B}} \sqrt{2 \pi} \sigma} \exp \left[-\frac{1}{2 \sigma^{2}} \ln ^{2}\left(\frac{T_{\mathrm{B}}}{\left\langle T_{\mathrm{B}}\right\rangle}\right)\right],
$$

where $\left\langle T_{\mathrm{B}}\right\rangle, \sigma$, and $A$ denote a mean blocking temperature, distribution width, and amplitude of the distribution, respectively.

The line in Fig. 4(d) was obtained fitting the ZFC curve according to Eq. (3). At low temperatures, we observed the decreasing slopes, but they were not well fitted with Eq. (3), thus reflecting they are unlikely to be a distribution $f\left(T_{\mathrm{B}}\right)$. From this picture, we note that there is no $f\left(T_{\mathrm{B}}\right)$ for the unirradiated sample in this temperature regime. Before the irradiation, the sample thus stays in the blocked regime, indicating $T_{\mathrm{B}}$ would be over $300 \mathrm{~K}$. After the beam irradiation, however, a distribution $f\left(T_{\mathrm{B}}\right)$ at $201 \mathrm{~K}$ that was well fitted with Eq. (3) arises from a fraction of particles exhibiting superparamagnetic behavior, in agreement with a separated distribution of the particles (Fig. 2(b)). It is supported by the unsaturated magnetization in the M-H curve at $300 \mathrm{~K}$ of Fig. 3(b). We thus suggest that the coexistence of superparamagnetism and spin-glass behavior may explain this anomalous magnetic behavior. In other words, both the magnetic frozen regime and dynamic regime may be present in this temperature regime, making interparticle interaction possible. As a result, a long-range magnetic structure specific to dipolar interaction between neighboring particles may enhance the $M_{\mathrm{S}}$ as displayed in Fig. 3(b).

\section{Conclusions}

In summary, we have investigated the magnetism in the CI powders before and after the $\mathrm{Ni}^{+}$ion beam irradiation. Unexpectedly, we found that the particle distribution becomes broader up to $\sim 4 \mu \mathrm{m}$ after the irradiation, from up to $\sim 1.5 \mu \mathrm{m}$ before the irradiation. Furthermore, after the irradiation, the distribution was well fitted with two log-normal distribution functions, unlike a one distribution function before irradiation. In $\mathrm{M}-\mathrm{H}$ data, ferromagnetic hysteresis curves were observed for both samples, but with substantially increased saturation magnetization. To understand this anomalous magnetic behavior, we measured the ZFC and FC curves as a function of temperature, and thus found that the spin-glass-like behavior arising from spin frustration in the intraparticle may enhance the magnetization with increasing temperature. After the irradiation, the presence of the separated distribution of blocking temperature may be ascribed to the fraction of magnetically interacting particles. Therefore, the Ni ion introduced CI powders induces the magnetic interaction between neighboring particles in a frustrated spin system, and thus increases the saturation magnetization.

\section{Methods}

Pure CI powder (grade EW; BASF, Germany) was used as-received and consists of particles with a diameter of 0.4-1.5 $\mu$ m. To irradiate the $\mathrm{Ni}^{+}$ion beam onto the samples, we use an accelerator with the beam energy of $140 \mathrm{keV}$. The irradiated dose of $\mathrm{Ni}^{+}$beam was $5 \times 10^{16} \mathrm{~cm}^{-2}$. During the irradiation, the sample was kept at room temperature using a cooling system. The structure of CI powders before and after irradiation was examined by X-ray diffraction (XRD). The chemical state of the 
surface for the samples was analyzed by an X-ray photoelectron spectrometry (XPS). The size and shape of the particles were examined using a scanning electron microscopy (SEM) (JEOL, Tokyo, Japan). The distribution of the particle sizes of the CI powders before and after irradiation was examined by a dynamic light scattering (DLS) analysis. Inductive coupled plasma mass spectroscopy (ICP-MS) was carried out to measure the element concentration of $\mathrm{Ni}$ in CI powder. The magnetization curves were obtained from a superconducting quantum interference device (SQUID) at Korea Basic Science Institute (KBSI).

\section{References}

1. Wang, H., Zhu, D., Zhou, W. \& Luo, F. Elecromagnetic and microwave absorption properties of the carbonyl iron/TiC hybrid powders in the X band. Int. J. Magnetics Electromagnetism 2, 005 (2016).

2. Zhang, Z., Liu, X., Zhang, H. \& Li, E. Electromagntic and microwave absorption properties of carbon fibers coated with carbonyl iron. J. Mater. Sci: Mater. Electron. 26, 6518-6525 (2015).

3. Pan, Y. et al. Electromagnetic and microwave absorption properties of coatings based on spherical and flasky carbonyl iron. J. Mater. Sci: Mater. Electron. 30, 18123-18134 (2019).

4. Ge, C. et al. Electromagnetic and microwave absorption properties of iron pentacarbonyl pyrolysis-synthesized carbonyl iron fibers. $R S C A d v$. 10, 23702 (2020).

5. Plachy, T. et al. Impact of corrosion process of carbonyl iron particles on magnetorheological behavior of their suspensions. J. Ind. Eng. Chem. 66, 362-369 (2018).

6. Martínez-Boubeta, C. et al. Critical radius for exchange bias in naturally oxidized Fe nanoparticles. Phys. Rev. B 74, 054430 (2006).

7. Klygach, D. S. et al. Magnetic and microwave properties of carbonyl iron in the high frequency range. J. Magn. Magn. Mater. 490, 165493 (2019).

8. Guo, J., Duan, Y., Liu, L., Chen, L. \& Liu, S. Electromagnetic and microwave absorption properties of carbonyl-iron/Fe ${ }_{91} \mathrm{Si}_{9}$ composites in gigahertz range. JEMAA 3, 140-146 (2011).

9. Molina-Concha, B., Zysler, R. D. \& Romero, H. Anomalous magnetization enhancement and frustration in the internal magnetic order on $\left(\mathrm{Fe}_{0.69} \mathrm{Co}_{0.31}\right) \mathrm{B}_{0.4}$ nanoparticles. Appl. Sci. 2, 315-326 (2012).

10. Fernández-García, M. P. et al. Co nanoparticles inserted into a porous carbon amorphous matrix: the role of cooling field and temperature on the exchange bias effect. Phys. Chem. Chem. Phys. 13, 927-932 (2011).

11. Li, S., Liu, R. \& Xiong, X. Preparation and characterization of carbonyl iron soft magnetic composites with magnesioferrite insulating coating layer. Trans. Nonferrous Met. Soc. China 30, 3067-3077 (2020).

12. Li, D. Y. et al. Relaxor ferroelectricity and magnetoelectric coupling in ZnO-Co nanocomposites thin films: beyond multiferroic composites. ACS Appl. Mater. Interfaces 6, 4737-4742 (2014).

13. Denardin, J. C. et al. Thermoremanence and zero-field-cooled/field-cooled magnetization study of $\mathrm{Co}_{x}\left(\mathrm{SiO}_{2}\right)_{1-x}$ granular films. Phys. Rev. B 65, 064422 (2002).

14. Nunes, W. C., Folly, W. S. D., Sinnecker, J. P. \& Novak, M. A. Temperature dependence of the coercive field in singledomain particle systems. Phys. Rev. B 70, 014419 (2004).

15. Greculeasa, S. G. et al. Tuning structural and magnetic properties of Fe oxide nanoparticles by specific hydrogenation treatments. Sci. Rep. 10, 17174 (2020).

16. Alzoubi, G. M. et al. Coexistence of superparamagnetism and spin-glass like behavior in zinc-substituted cobalt ferrite nanoparticles. Appl. Phys. A 26, 512 (2020).

17. Fabris, F., Tu, K.-H., Ross, C. A. \& Nunes, W. C. Influence of dipolar interactions on the magnetic properties of superparamagnetic particle systems. J. Appl. Phys. 126, 173905 (2019).

18. Umashankar, S. et al. Competing magnetic interactions and superparamagnetism like behavior in $\mathrm{xNiFe}_{2} \mathrm{O}_{4}-(1-\mathrm{x}) \mathrm{BaTiO}_{3}$ (x=0.2 and 0.3) nano composites. J. Magn. Magn. Mater. 439, 213-219 (2017).

19. Livesey, K. L. et al. Beyond the blocking model to fit nanoparticles ZFC/FC magnetization curves. Sci. Rep. 8, 11166 (2018).

20. Biasi, E. D., Ramos, C. A. \& Zysler, R. D. Large surface magnetic contribution in amorphous ferromagnetic nanoparticles. Phys. Rev. B 65, 144416 (2002).

21. Nunes, W. C. et al. Role of magnetic interparticle coupling on the field dependence of the superparamagnetic relaxation time. Phys. Rev. B 72, 212413 (2005). 
22. Singh, P., Shukla, M. \& Upadhyay, C. Signatures of consolidated superparamagnetic and spin-glass behavior in magnetitesilver core-shell nanoparticles. Nanoscale 10, 22583 (2018).

23. Sato, S., Uchida, Y. \& Tamura, R. Spin symmetry breaking: superparamagnetic and spin glass-like behavior observed in rod-like liquid crystalline organic compounds contacting nitroxide radical spins. Symmetry 12, 1910 (2020).

24. Concha, B. M., Biasi, E. D. \& Zysler, R. D. Monte Carlo simulation of Fe-Co amorphous nanoparticles magnetization. Physica B 403, 390-393 (2008).

25. Vasundhara, K. et al. Size dependent magnetic and dielectric properties of nano $\mathrm{CoFe}_{2} \mathrm{O}_{4}$ prepared by a salt assisted gel-combustion method. J. Appl. Phys. 113, 194101 (2013).

\section{Acknowledgements}

This work was supported by the National Research Foundation of Korea (NRF) grant (NRF-2020M3F6A1081012) and KOMAC operation fund of KAERI by Ministry of Science ICT and Future Planning of Korean Government. The measurements at the Korean Basic Science Institute (KBSI) are acknowledged.

\section{Author contributions}

J.K.P. and W.-J.C. contributed to the conception and design of the work; H.M.J., D.-S.K, and J.S.L. planned and designed the experiments, and made data acquisition; C.K., J.S., and J.S.L. designed and made the beam-irradiation experiments; W.-J.C. and H.M.J contributed to design of the sample preparation; J.K.P and J.S. contributed to analysis, data interpretation. All authors wrote and reviewed the manuscript. J.K.P. supervised the project.

\section{Additional information}

The authors declare no competing financial interests. 


\section{Figure Legends}

Figure 1: (a) XRD pattern of the unirradiated and the $\mathrm{Ni}^{+}$ion irradiated CI powders. The inset shows the SEM images of the size and surface morphology of the unirradiated CI particles. XPS spectra of Fe $2 p$ for (b) the unirradiated and (c) the irradiated CI powders.

Figure 2: Particle size histograms (bars) obtained from the DLS analysis for (a) the unirradiated and (b) $\mathrm{Ni}^{+}$-beam irradiated CI powders, and the fits of the data according to a log-normal distribution. Unlike the unirradiated sample, the irradiated sample was well fitted with two superposed distribution functions. The fits give the median particle diameter $\langle D\rangle=0.782 \pm 0.002$ $\mu \mathrm{m}$ and the distribution width $\sigma_{D}=8.66 \pm 0.09$ for the unirradiated sample, and $\left\langle D_{1}\right\rangle=1.962 \pm 0.009 \mu \mathrm{m}, \sigma_{D_{1}}=8.50 \pm 0.16$, $\left\langle D_{2}\right\rangle=1.698 \pm 0.068 \mu \mathrm{m}, \sigma_{D_{2}}=3.35 \pm 0.08$ for the irradiated sample.

Figure 3: Magnetization curves of (a) the unirradiated and (b) the $\mathrm{Ni}^{+}$ion irradiated $\mathrm{CI}$ powders measured at $300 \mathrm{~K}$ and 5 $\mathrm{K}$ by using SQUID. The inset of each figure shows the curve measured at the lower magnetic field.

Figure 4: ZFC-FC magnetization curves of (a) the unirradiated and (b) the $\mathrm{Ni}^{+}$ion irradiated CI powders measured with an applied magnetic field of $500 \mathrm{Oe}$. Blocking temperature distribution obtained from the derivative $d\left(M_{\mathrm{ZFC}}-M_{\mathrm{FC}}\right) / d T$ of the experimental data for (c) the unirradiated and (d) the irradiated samples. In Fig. 4(d), the red solid line is a fit of the data according to Eq. (3). 

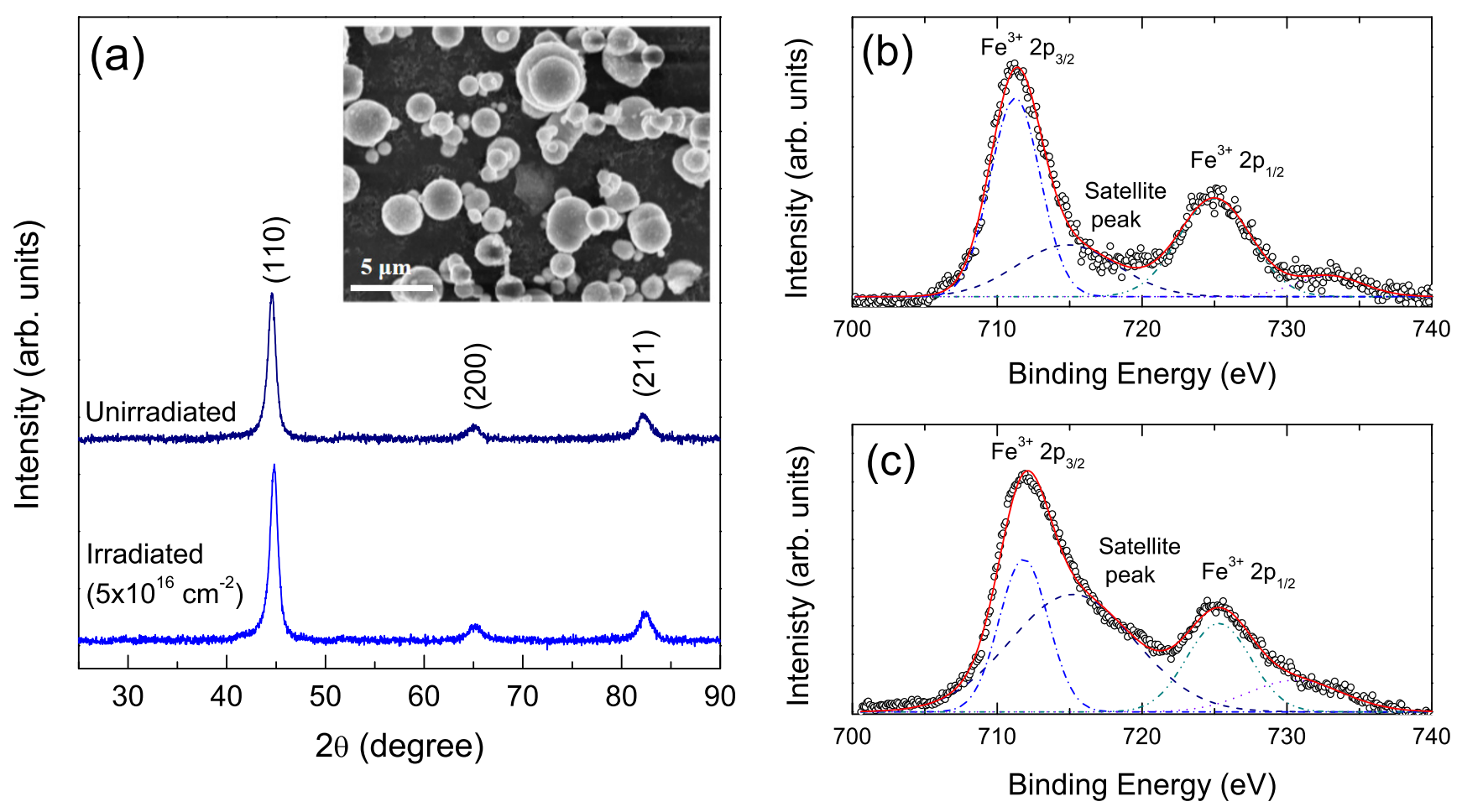

Figure 1 

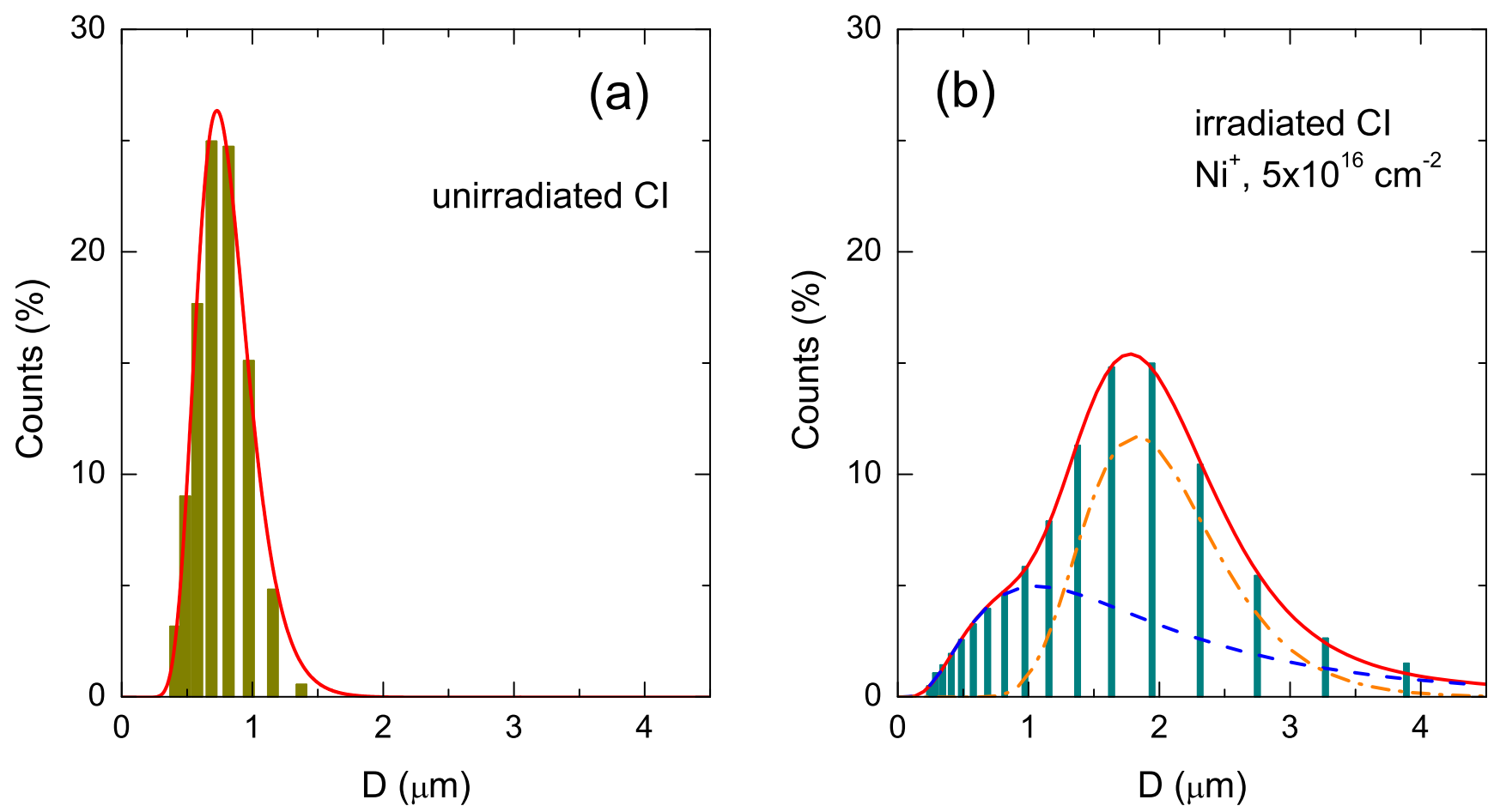

Figure 2 

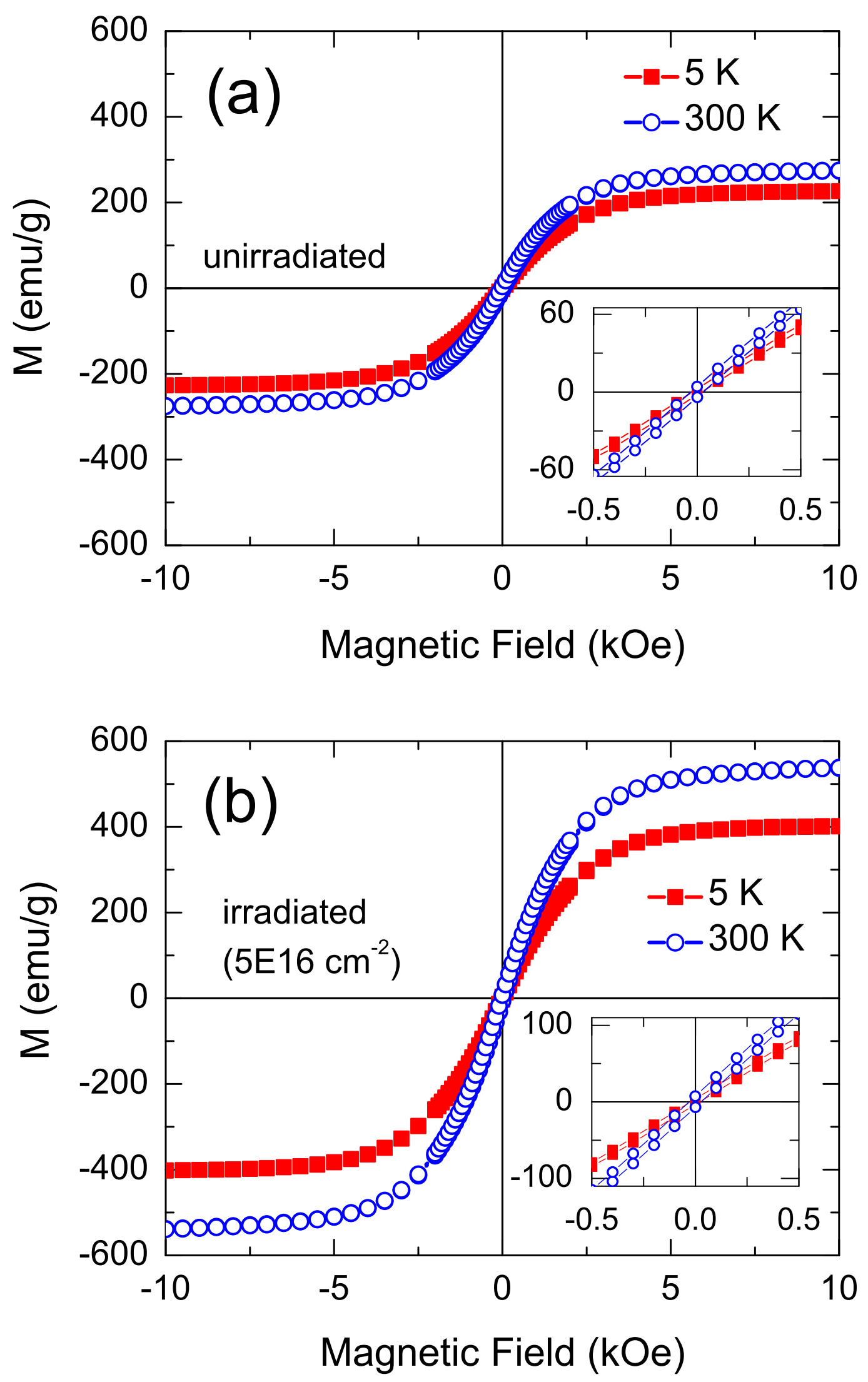

Figure 3 

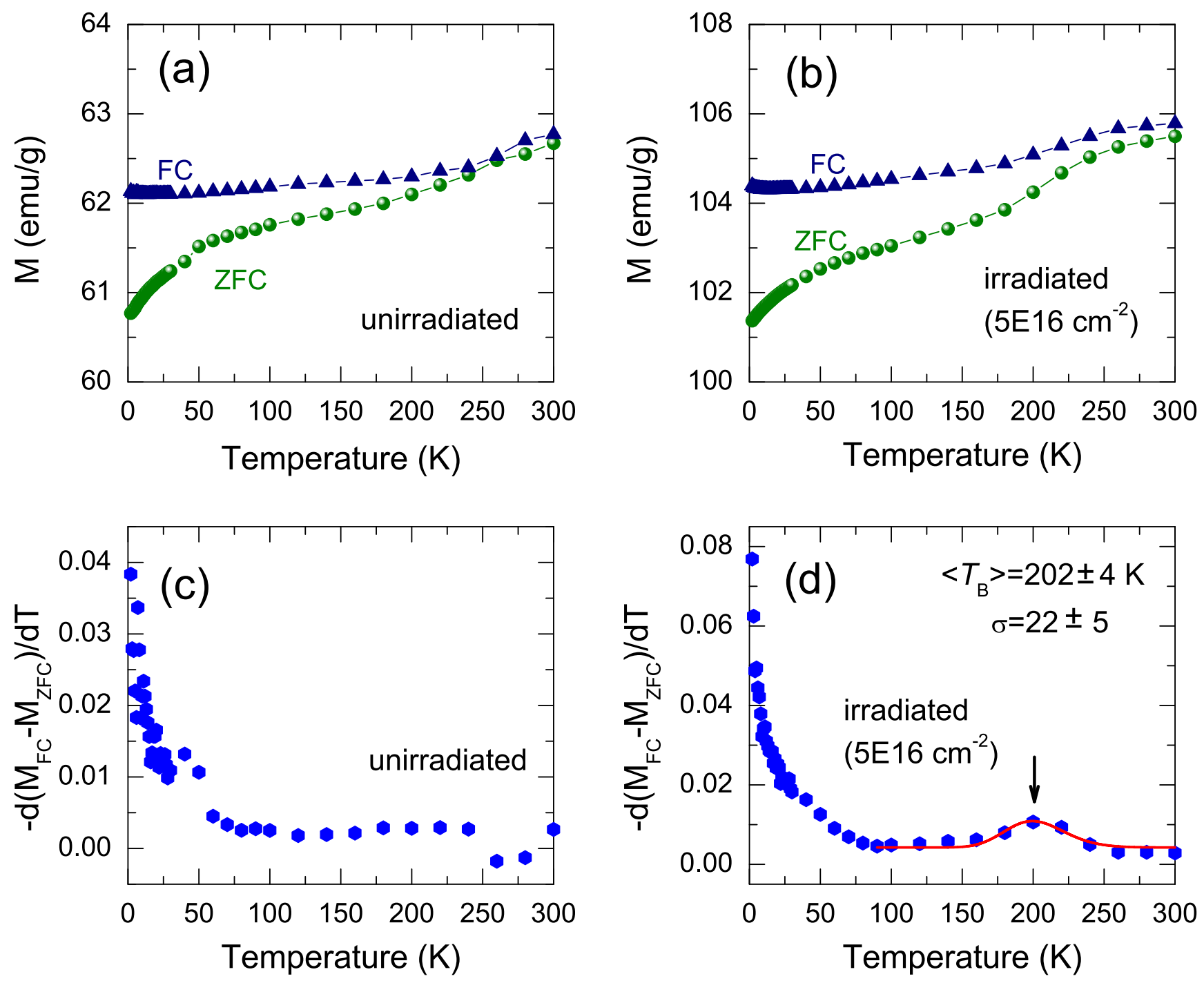

Figure 4 